\title{
Low and Delayed Recruitment at Two Grey Seal Breeding Colonies in the UK
}

\author{
P. Pomeroy, S. Smout and S. Moss \\ NERC Sea Mammal Research Unit, Gatty Marine Laboratory, University of St Andrews, \\ St Andrews KY16 8LB, UK. \\ E-mail: pp6@st-andrews.ac.uk \\ S. Twiss \\ School of Biological and BioMedical Sciences, South Road, \\ The University of Durham, Durham \\ DH1 3LE, UK. \\ R. King \\ School of Mathematics and Statistics, Mathematical Institute, \\ North Haugh, St Andrews, Fife KY16 9SS, UK.
}

Pomeroy, P., S. Smout, S. Moss, S. Twiss, and R. King. 2010. Low and delayed recruitment at two grey seal breeding colonies in the UK. J. Northw. Atl. Fish. Sci., 42: 125-133. doi:10.2960/J.42.m651

\begin{abstract}
This study is concerned with changes in the recruitment of UK grey seal pups into the adult breeding population. Pups were marked over two decades at North Rona and the Isle of May. The proportion of these animals observed to recruit as adults was at best 0.10 on North Rona and 0.31 on the Isle of May. Double-tagged cohorts were re-sighted at the highest rates, compared with cohorts that were marked with single tags or with brands. There was also evidence of substantial interannual variation, and no individuals were ever re-sighted for certain cohorts. Estimates of absolute tag loss were higher at North Rona than at the Isle of May, but not sufficient to explain the low re-sight rates there. Recruitment at the Isle of May appears to be occurring later in recent years and this is consistent with the effects of density dependence. There are too few tag returns from North Rona to allow the investigation of any time-dependence in recruitment, but this lack and the continued decline of pup production on North Rona suggests that recruitment there may be low. These findings have direct implications for models of UK grey seal population dynamics.

Keywords: density dependence, grey seal, Halichoerus grypus, Isle of May, North Rona, pup production, recruitment, tags
\end{abstract}

\section{Introduction}

The status and dynamics of marine mammal populations offer valuable information about their potential role as top predators within the marine environment. The population status of UK grey seals is monitored annually by means of large-scale pup production surveys carried out during the breeding season. While these surveys provide an index of pup production and thus of the number of breeding female seals, estimates of total population (including males, immature animals and non-breeding females) require population models in which vital rates are critical. Recently, effort has been directed at developing such models within a Bayesian framework. Using these models to predict total population size depends strongly on the assumptions that are made about the biological mechanisms driving density dependence and whether these derive from fecundity or mortality (Buckland et al., 2007). Such models are informed by empirical studies and this is achieved conveniently by using informative priors.

Much of the basic life history information available for grey seals in the UK was collected through synoptic lethal sampling in the 1960s, 1970s and 1980s when the population was increasing exponentially (Hewer, 1964; Boyd and Campbell, 1971). These data suggested that females recruited into the breeding population aged 3-5 and that by age 5 , over $90 \%$ of females would be breeding. Recently however, the UK grey seal population's annual overall rate of pup production has declined from more than $+6 \%$ in the 1990 s to $+0.5 \%$ in 2004 (Duck and MacKey, MS 2005). Therefore it is clear that demographic 
parameters have changed for the population as a whole, but there have been substantial variations within this trend in different geographic areas. In the Outer Hebrides on the UK's west (Atlantic) coast, pup production has declined steadily, while on the east (North Sea) coast, production has increased. Contrasts in the population trajectories in these areas might be due to differences in adult mortality and fecundity, sub-adult survival and recruitment, or a combination of factors. Recent results indicate that migration between these large-scale areas is not likely to be important (Duck and Mackey, MS 2008).

In this study, we present empirical evidence of varying recruitment of female grey seals, using markrecapture data collected during long-term reproductive studies at two geographically separated and contrasting breeding colonies to offer insights into these demographic changes and how they might vary. In order to correct for a potential bias in estimates of recruitment, we also estimate tag loss rates.

\section{Methods}

North Rona (NR) in the Outer Hebrides, and Isle of May (IoM) in the Firth of Forth are separated by approximately 200 miles (Fig. 1). Annual pup production at NR, monitored by means of annual aerial surveys, has fallen from around 2500 in the 1960 s to around 1000 presently, while the IoM has grown from a handful of pups born in the 1970s to over 2000 in recent years (Fig. 2). Grey seals have been studied at these colonies by researchers present during the breeding season from the 1960s at NR, and at IoM from the 1980s. Work has continued with some interruptions to the present, providing a unique data set on individual animals from these two colonies. Such long-term studies are costly and labour-intensive, and have not been carried out at any other breeding colonies in the UK. However a Canadian colony at Sable Island has been intensively monitored (Bowen et al., MS 2007).

The initial focus of the studies was to determine colony-wide parameters, later shifting to individual-based studies, primarily on adults. The presence of marked individuals has been recorded systematically in every year that workers were present. Pups were also marked in some years. The return of such animals, once they recruited into the adult breeding population, could potentially be recorded provided that (1) the animals remained alive, recruited to their natal colony, and retained the marks that had been applied to them as pups, and (2) marked animals were identified during surveys of breeding females. Data presented here were gathered only at the

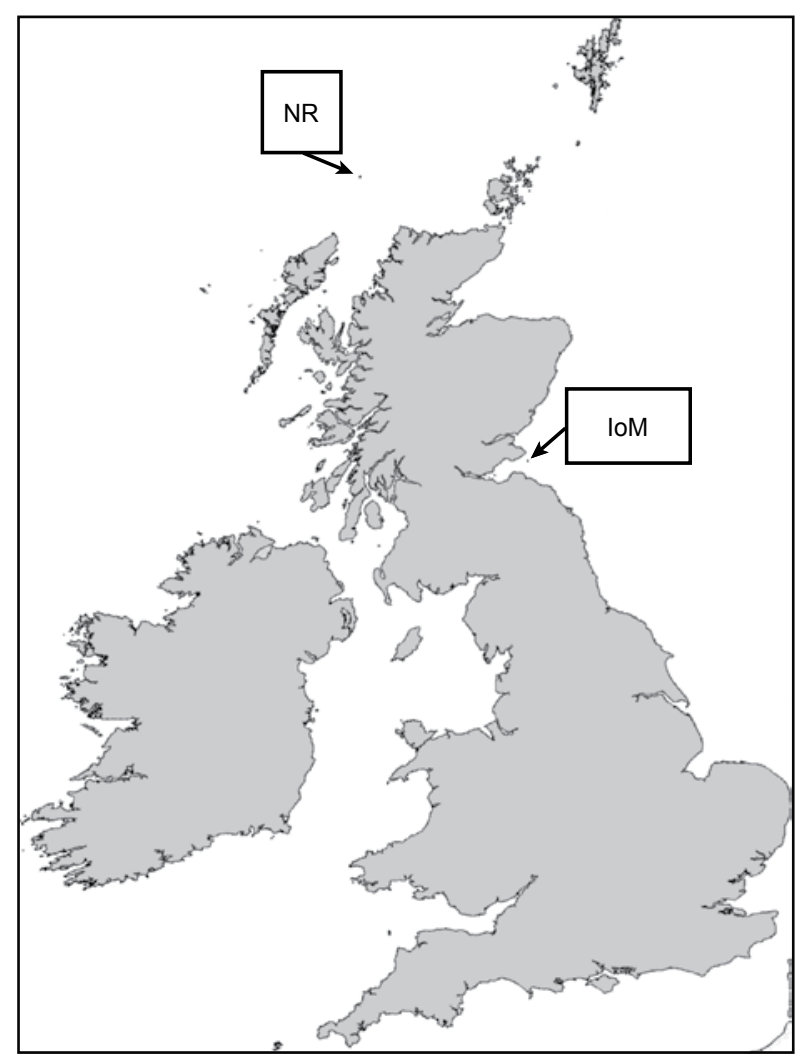

Fig. 1. The location of 2 UK grey seal breeding colonies: North Rona (NR) in the Outer Hebrides and the Isle of May (IoM) in the Firth of Forth, Scotland.

two natal colonies, thus animals that emigrated elsewhere were lost to the study.

The number and types of marks applied changed over time. Pups on NR were tail tagged in 1961-1962, cohort branded from 1960-1972, then tagged in the hind flippers with rototags (Dalton ID Systems Ltd., UK) during subsequent studies. On IoM, pups were tagged and only in 1990 were they also cohort branded. In 1991-1992, pups were double tagged. The tagging methods used during the long-term studies at both colonies are shown in Table 1. In 1995-1996, pups at both colonies were marked using subcutaneous RFID chips. These two cohorts are not considered here because it was found that these animals were not identifiable by sight in the field. On NR, re-sighting data was collected between 1960 and 2000, though observations were not made in all years (see Tables 1 and 2). On IoM, re-sightings were recorded from 1990 to 2005.

The terrain at NR and IoM, and the movement of animals to/from water sources, does not allow for groundbased censuses of all animals. At NR regular surveys of all visible seals were made during the breeding season. Observers surveyed these seals on foot using binoculars 


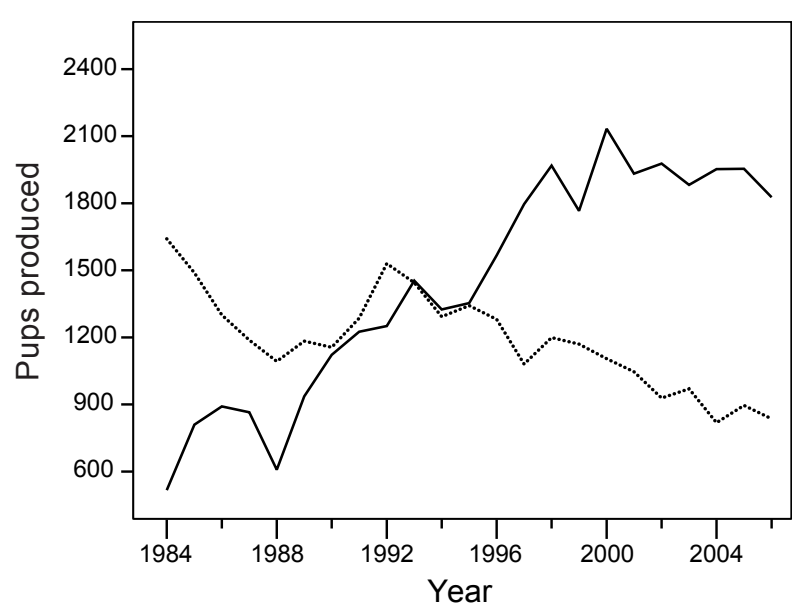

Fig. 2. Estimated pup production at North Rona (solid line) and the Isle of May (dotted line), based on annual aerial surveys.

and spotting scopes approximately every four days. Additional daily surveys of seals breeding in the southern half of the northern peninsula (the study area producing around half the island's pups) were carried out from hides overlooking the area during daylight hours. Capture work with individual seals afforded additional opportunities for closer inspection of animals in the study area. On IoM, observers carried out surveys with the aim of covering areas where animals were visible repeatedly, often daily, during the breeding season. These general surveys were supplemented by daily observations from hides overlooking very local parts of the colony in some years (Pomeroy et al., 1994, 1999, 2000). Observation effort at both sites did not decrease over the study period, and if anything it has increased, but observation effort will not have been constant from year to year because the identity of observers changed, and the focus of some of the observational studies undertaken at the colonies also changed over time.

Any tagged animals present were identified as practicable, either from a distance with the aid of telescope or binoculars or close up by direct inspection. However, flipper tags cannot always be read by observers even when they are known to be present, e.g. if the codes are obscured by dirt or the animal is orientated in such a way that tags cannot be seen clearly. The percentage of tagged seals (estimated from observations of tagged animals compared with positive identifications of individuals) that were identified during regular surveys was estimated typically as c. $90 \%$ on NR and c. $70 \%$ on IoM. We use "re-sight" to mean a definite sighting of a particular animal (tag/s read). Where animals had originally been double-tagged, the status of both tags (present or absent) were recorded where possible.
TABLE 1. Summary of tags applied to grey seal pups at North Rona and Isle of May and cumulative percentage re-sights of these up to 2006. If $m$ was the number of seals from year $t$ seen by 2006 , and $n$ was the number marked as pups in year $t$, then the cumulative percentage re-sight rate is $100 \mathrm{~m} / \mathrm{n}$.

\begin{tabular}{|c|c|c|c|c|c|}
\hline \multirow[b]{2}{*}{ Year } & \multicolumn{3}{|c|}{ Total pups } & \multicolumn{2}{|c|}{ Females re-sighted } \\
\hline & Tagged & Males & $\overline{\text { Females }}$ & Number & $\%$ \\
\hline \multicolumn{6}{|c|}{ North Rona flipper and tail tagging } \\
\hline 1960 & 223 & 114 & 109 & 0 & 0.0 \\
\hline 1961 & 541 & 294 & 247 & 0 & 0.0 \\
\hline $1962-1978$ & \multicolumn{3}{|c|}{ no tagging } & & \\
\hline 1979 & 20 & 9 & 11 & 1 & 9.1 \\
\hline 1980 & 500 & 255 & 245 & 0 & 0.0 \\
\hline 1981 & 20 & 9 & 11 & 1 & 9.1 \\
\hline- & - & - & - & - & \\
\hline 1985 & 82 & 41 & 41 & 3 & 7.3 \\
\hline 1986 & 208 & 116 & 92 & 5 & 5.4 \\
\hline 1987 & 46 & 25 & 21 & 1 & 4.8 \\
\hline 1988 & 20 & 10 & 10 & 1 & 10.0 \\
\hline 1989 & 33 & 17 & 16 & 1 & 6.3 \\
\hline- & - & - & - & - & \\
\hline 1993 & 296 & 152 & 144 & 4 & 2.8 \\
\hline 1994 & 301 & 161 & 140 & 0 & 0.0 \\
\hline 1995 & $*$ & $*$ & $*$ & $*$ & $*$ \\
\hline 1996 & $*$ & $*$ & $*$ & $*$ & $*$ \\
\hline 1997 & 322 & 166 & 156 & 0 & 0.0 \\
\hline 1998 & 108 & 57 & 51 & 0 & 0.0 \\
\hline 1999 & 71 & 36 & 35 & 0 & 0.0 \\
\hline 2000 & 40 & 17 & 23 & 0 & 0.0 \\
\hline $\begin{array}{l}\text { Total } \\
\text { post-1978 }\end{array}$ & 2067 & 1071 & 996 & $17^{1}$ & 1.7 \\
\hline
\end{tabular}

\begin{tabular}{lrrrrr}
\hline Isle of May flipper tagging & & & \\
1990 & 139 & 70 & 69 & 15 & 21.7 \\
1991 & 174 & 87 & 87 & 27 & 31.0 \\
1992 & 171 & 92 & 79 & 11 & 13.9 \\
1993 & 353 & 177 & 176 & 14 & 8.0 \\
1994 & 369 & 175 & 194 & 17 & 8.8 \\
1995 & $*$ & $*$ & $*$ & $*$ & $*$ \\
1996 & $*$ & $*$ & $*$ & $*$ & $*$ \\
1997 & 521 & 263 & 258 & 4 & 1.6 \\
1998 & 96 & 45 & 51 & 1 & 2.0 \\
1999 & 140 & 65 & 75 & 5 & 6.7 \\
2000 & 29 & 15 & 14 & 0 & 0.0 \\
2001 & 147 & 84 & 63 & 0 & 0.0 \\
2002 & 195 & 102 & 93 & 0 & 0.0 \\
2003 & 93 & 41 & 52 & 0 & 0.0 \\
2004 & 59 & 30 & 29 & 0 & 0.0 \\
2005 & 32 & 12 & 20 & 0 & 0.0 \\
\hline Total & 2518 & 1258 & 1260 & 94 & 7.5 \\
\hline
\end{tabular}

* indicates years in which no visible tags were applied.

${ }^{1}+1$ Shetland tagged pup 
TABLE 2. Summary of brands applied to grey seal pups at North Rona and re-sights of these between 1978 and 1998. The number of animals expected to be alive in years subsequent to branding was based on assumed first year survival of 0.62 with survival in subsequent years fixed at 0.95 .

\begin{tabular}{|c|c|c|c|c|c|c|c|}
\hline Cohort & $\begin{array}{c}\text { Females } \\
\text { marked }\end{array}$ & 1978 & 1981 & 1986 & 1989 & 1995 & 1998 \\
\hline \multicolumn{8}{|c|}{ Re-sights } \\
\hline 1960 & 102 & 0 & 0 & 0 & 0 & 0 & 0 \\
\hline 1961 & 278 & 0 & 0 & 1 & 0 & 0 & 0 \\
\hline 1962 & 300 & 2 & 0 & 3 & 1 & 2 & 0 \\
\hline 1963 & 302 & 2 & 1 & 3 & 2 & 2 & 1 \\
\hline 1964 & 270 & 6 & 3 & 7 & 8 & 6 & 2 \\
\hline 1965 & 346 & 6 & 3 & 4 & 7 & 5 & 3 \\
\hline 1966 & 300 & 5 & 2 & 5 & 4 & 3 & 2 \\
\hline 1967 & 201 & 5 & 3 & 3 & 1 & 2 & 1 \\
\hline 1968 & 236 & 7 & 3 & 3 & 3 & 1 & 1 \\
\hline \multicolumn{8}{|c|}{ Number expected alive } \\
\hline 1960 & 102 & 26 & 23 & 18 & 15 & 11 & 9 \\
\hline 1961 & 278 & 76 & 65 & 50 & 43 & 32 & 27 \\
\hline 1962 & 300 & 86 & 74 & 57 & 49 & 36 & 31 \\
\hline 1963 & 302 & 91 & 78 & 61 & 52 & 38 & 33 \\
\hline 1964 & 270 & 86 & 74 & 57 & 49 & 36 & 31 \\
\hline 1965 & 346 & 116 & 99 & 77 & 66 & 48 & 42 \\
\hline 1966 & 300 & 106 & 91 & 70 & 60 & 44 & 38 \\
\hline 1967 & 201 & 75 & 64 & 50 & 42 & 31 & 27 \\
\hline 1968 & 236 & 92 & 79 & 61 & 52 & 39 & 33 \\
\hline
\end{tabular}

We examined tag loss in double marked animals. Arithmetic annual tag loss rates were calculated as the ratio of tags lost to tag years and compared to tag loss rates generated from (Cormack-Jolly-Seber based) models in which tag loss could be estimated by comparing the apparent survival of tagged versus branded animals (Smout et al., 2010).

We constructed a simple simulation model to estimate the expected number of females born at IoM and remaining alive and tagged from a given cohort in a given year. These expected values could then be compared to the observed data. Note that we assumed there was no emigration for our cohort.

The model can be written:

$$
N_{t+1}=\phi_{t}(1-\tau) N_{t}
$$

where $N_{t}$ is the number alive in year $t, \phi_{t}$, is the survival from year $t$ to $t+1$, and $\tau$ is the tag loss rate per annum.

Survival of animals was deterministic, and survival rates were based on realistic estimates of seal life history parameters from independent studies. We set female first-year survival at 0.62 (Hall et al., 2001), 0.52 and 0.48 to cover a range of plausible values. We assumed survival rates of 'adult' females (those in their second year and older) to be consistent at 0.95 thereafter (Harrison et al., 2006). The effects of different plausible values of tag loss rate were also included.

We then assumed that differences between observed recruitment and our predictions could potentially be accounted for by several alternative mechanisms including mortality rates and tag loss rates that are outside the ranges we have assumed, or emigration of pups to other colonies.

\section{Results}

\section{Re-sights at NR: flipper tags}

For a given cohort of animals that were marked individually as pups, we use the term 'cumulative re-sight rate' to indicate the proportion of different individuals in a marked cohort that were subsequently re-sighted as adults on the colony on one or more occasions. Subadults are unlikely to be present on the breeding colony, so cumulative re-sight rate for a given cohort is expected to increase with time initially, tending to an asymptotic value when most of the adults that have survived (and retained their mark) to recruit into the breeding population have been sighted. The cumulative re-sight rate for a given cohort and year is equivalent to the probability that, in that year, an animal marked as part of the cohort has been seen again as an adult at the colony on at least one occasion.

Cumulative re-sight rates at NR were, at most, $10 \%$ (Table 1). There were no re-sights of any pups tail-tagged in 1961 and 1962. Tail tags were deemed a failure as they were sometimes lost before weaning (Boyd and Campbell, 1971). Re-sights from other NR cohorts were sparse, even though approximately $25 \%$ of female pups were tagged in some years. There have been no re-sights to date of any of the 400 NR female pups flipper-tagged in 1994 and from 1997-2000.

One female breeding on NR in 1995 and not seen since had been tagged as a 1977 pup in Shetland. An adult female branded on the Monach Isles in summer 1996 has since bred regularly on NR.

Based on a total of 731 individuals tagged as pups between 1978 and 1994 and subsequently re-sighted there, the median age of females when they were first seen breeding on NR was 10, range 7-18 $(n=18)$. Because data 
for most cohorts were sparse, it was difficult to explore any possible changes in age at primiparity over time, and we do not present any such analysis for the NR data.

The annual tag loss rate for adult females at NR was estimated as $0.04(35 / 832)$, which is in agreement with the estimate generated for adult females using CormackJolly-Seber based models to estimate survival and tag loss rates of $4.2 \%$ ( $95 \%$ credible interval: $0.4 \%, 9.2 \%$ ).

\section{Re-sights at NR: pup cohort brands}

Year-specific brands applied to pups in the 1960s meant that up to $30 \%$ of the female pups produced were marked before they left the colony. There was extreme variability in re-sighting rates for branded animals (Table 2). For example, none of the 380 female pups branded in 1960-1961 were ever re-sighted. The re-sight rate for all branded cohorts in the NR breeding colony was very low, rarely representing more than $10 \%$ of the number of branded pups expected to be alive at any stage. None of these animals have been seen on NR since 1998 .

\section{Re-sights at IoM}

Cumulative re-sight rates to 2005 represent up to $31 \%$ of the females marked per cohort as pups at IoM (Table 1). The highest re-sight rates are from the 1990, 1991 and 1992 cohorts, the latter two of which were double marked.

From 1993 onwards, cumulative re-sight rates appear to be lower. Fewer than $10 \%$ of the marked pups from these cohorts have been re-sighted breeding on IoM. No females from the 2000 or later cohorts have been resighted on IoM to date.

As at NR, the number of re-sights obtained is not a simple function of the number of tags applied. Cumulative re-sight rates are variable, and some of the largest tagged cohorts have not had any re-sights.

The youngest animals observed breeding were age 4 and 5, but there were few observed below age 6 . A large proportion of females were not observed at the colony till they reached the age of 10 or more (Fig. 3). The mean age at which seals were first sighted, based on a total of 89 animals (pooled over all cohorts) was 9.4 years $(s d=2.7$ ). It is clear that this tendency for animals to be observed recruiting late is not confined to the earlier 1990-1992 cohorts but is as pronounced in the 1993, 1994 and 1997 cohorts (Fig. 3).

Annual tag loss rate at IoM was estimated as $2 \%$

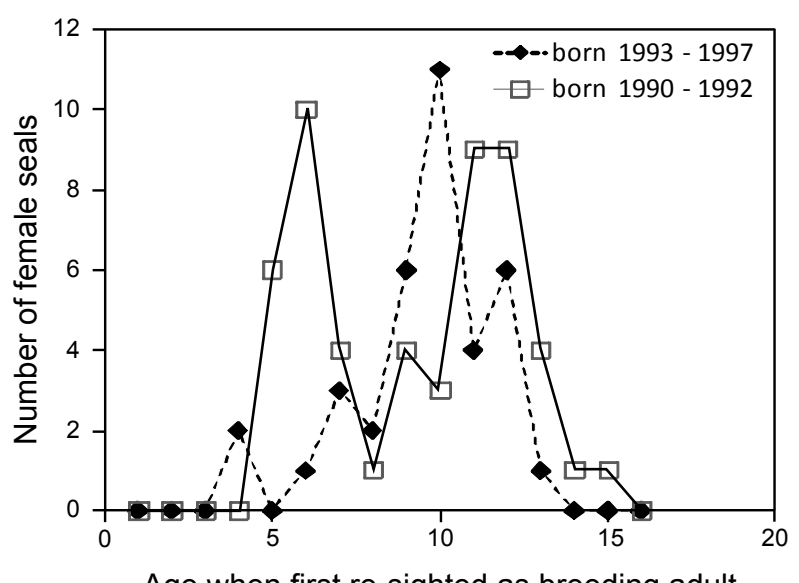

Age when first re-sighted as breeding adult

Fig. 3. Distribution of the age at which female seals at the Isle of May that had been marked as pups were first re-sighted as breeding adults. The dashed line represents cohorts born from 1993 to1997. The solid line represents cohorts born in 1990, 1991 and 1992.

(31/1406), while Cormack-Jolly-Seber based models fitted to data on adult animals using a Bayesian approach produced a higher estimate of $11 \%(95 \%$ credible interval $3.5 \%, 18 \%$ ) (Smout et al., 2007).For further detailed discussion of Bayesian approaches (with particular reference to Cormack-Jolly-Seber models and credible intervals see King et al., 2009).

The number of females known to be alive (Fig 4, green curves) and cumulative re-sights (red curves) suggest that in 1990-1991 the 10\% tag loss rate seems high (compare with black curves). However, in 1992 and subsequently to 1997 , re-sights were even lower than predicted by any of the model survival and tag loss rates. Note that in these parameter ranges, the effects of different first year survival rates are subtle after the first few years, compared to different tag loss rates: the predicted number of surviving tagged seals in a given year is more sensitive to the value chosen for tag loss rate than it is to the value chosen for first year survival, within the plausible ranges that were explored in the analysis.

\section{Discussion}

The study found quite different patterns of recruitment at the two colonies although similar methods were used at both sites, and similar numbers of animals had been marked. NR and IoM cannot be considered to be closed populations, in contrast for example to the well-studied breeding population at Sable Island in Canada (Bowen et al., 2007). Therefore the meaning of the term 'recruitment' here 

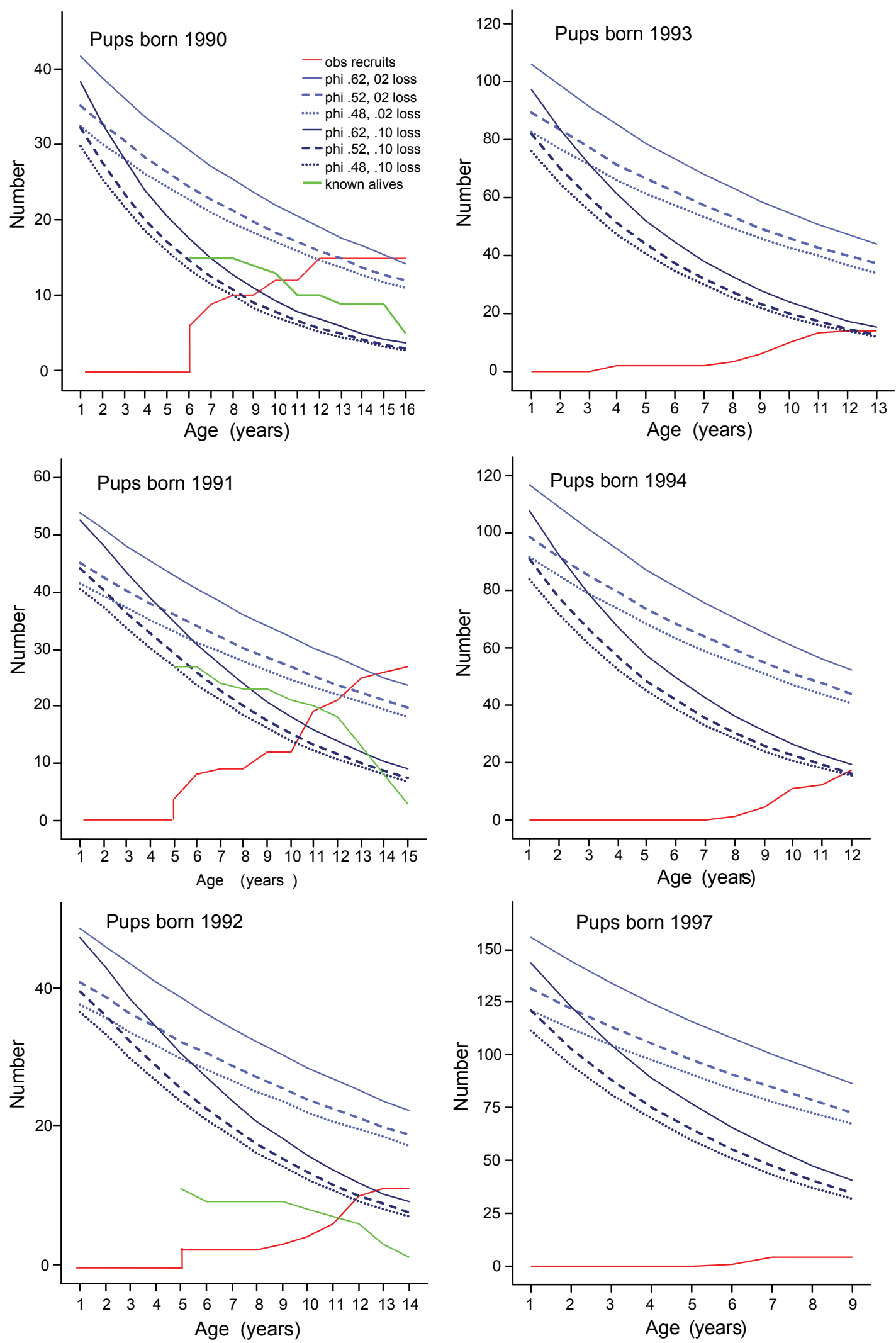

Fig. 4. The number of females known to be alive (Fig 4, green curves) and cumulative re-sights (red curves) suggest that in 1990-1991 the 10\% tag loss rate seems high (compare with black curves). However, in 1992 and subsequently to 1997, resights were even lower than predicted by any of the model survival and tag loss rates. Note that in these parameter ranges, the effects of different first year survival rates are subtle after the first few years, compared to different tag loss rates: the predicted number of surviving tagged seals in a given year is more sensitive to the value chosen for tag loss rate than it is to the value chosen for first year survival, within the plausible ranges that were explored in the analysis. 
should perhaps be clarified: in our study, recruitment indicates the return of animals that were observed as pups to breed in visible areas of the study colonies. Individual animals might emigrate to other colonies or even to outlying regions of the study colony, and if so they would not have been recorded as recruits.

The complete absence of some cohorts from re-sights has several possible explanations including: (1) mark loss (2) complete failure of the cohort through catastrophic mortality caused by e.g. disease, starvation, environmental effects, and (3) emigration of a cohort from the natal site, or recruitment of that cohort to an area of the colony that is particularly difficult to observe.

Mark loss was suspected in the case of NR tail tagged pups in 1960 and 1961, but does not explain the disappearance of the branded animals from the same years (brands generally remain visible for the lifetime of the animal). Some flipper-tagged cohorts, particularly at NR but also at IoM, have also been poorly represented. Therefore, severe fluctuations in early survival (or emigration) rates must remain a possibility. Because seals are long-lived animals and there is expected to be natural variation in age-at-recruitment, any signal from 'doomed' cohorts (or cohorts which recruit en masse to different colonies and therefore emigrate from the study site) is unlikely to be seen as a pulse in pup production at the natal colony. If early survival/recruitment shows a consistent downward trend, then a levelling off or decline in pup production would be expected but with a significant time lag from the onset of reduced pup survival, and there is the additional uncertainty that local recruitment might be counteracted by immigration from other breeding colonies.

Evidence to quantify permanent emigration and immigration by immature animals at the UK colonies is largely lacking. This would require either longterm telemetry studies on many animals, or long-term mark-recapture work at a large number of sites. These approaches are not currently feasible though advances in technology might in future allow for such studies. Some suggestive evidence for movement between colonies does exist. Harwood et al. (1975) reported sightings of two NR cohort branded females on the Monach Isles and a breeding female on NR was tagged as a pup in Shetland. However, philopatry is estimated to be important at both study colonies, based on re-sightings of females observed to breed as adults at NR and IoM, and marked as pups. Permanent emigration by immature animals is likely to be limited, and previous estimates of emigration rates do not adequately explain the very low recruitment of pups observed in some cohorts (philopatry $\sim 40 \%$ at NR and $50 \%$ at IoM) (Allen et al., 1995; Pomeroy et al., 2000).
Since the 1990s, observation effort at both sites has been generally high and increasing overall (though not necessarily constant between years). Tagging has been carried out by the same people, often at both sites. Therefore it is difficult to attribute long-term colony differences in observed recruitment to differences either in the efficiency of re-sighting or the effectiveness of the tagging procedure.

There were few recruits of females marked as pups on NR as breeding adults. More recent re-sight rates for single tagged pups marked in the 1980s and 1990s were similar to the highest re-sight rates for those branded in the 1960s. It is unfortunate that the early branding programme was never followed up. Most of the data available on NR cohort branded animals from later studies refers to postrecruitment years and is difficult to interpret. However, the overall decline in the NR breeding colony has occurred at a rate which mirrors typical adult female mortality. If little or no recruitment was occurring there, adult mortality would account for the observed decline in pup production.

Some issues arise when choosing marking methods for studying recruitment of grey seals. First there is the problem that passive marks require observation and this is time and labour intensive. Photo-ID methods based on natural seal pelage markings are very effective in studies of adults (Smout et al., 2010) but currently cannot be used in pup recruitment studies because pelage markings change as pups mature. Work is in progress to address this problem. Telemetry devices require battery power which restricts their lifetime, and they must be attached with minimal impact. Branding studies have not been carried out recently either at IoM or NR. Existing cohort brands are very visible but they are non-specific, which does not allow for individual census of those present on a colony in subsequent years. Tagging is currently the chosen marking method for pup recruitment studies.

There has always been the suspicion that flipper tagged pups may suffer greater mortality in fishing gear. However, evidence is difficult to obtain from the recapture studies because such effects are entirely confounded with tag loss. If this does occur then, all other things being equal, there has been a stronger gear-specific effect on NR pups compared with those at IoM and this effect has also changed consistently with time at IoM (which we do not consider likely). Age-specific loss of tags may be a factor in this respect, but inevitably, passive tags must be retained for the years between weaning and breeding to be informative therefore losses in the intervening period are a problem.

Our results indicate that recruitment for animals at IoM now occurs later than was observed previously (Hewer, 1964; Boyd, 1985), and that this delay has 
become more pronounced with time. It should be noted that the first re-sights of individual females on the study colonies do not necessarily correspond to their age at primaparity: it is possible that an animal may not be observed in the first year in which it recruits, or for some years following recruitment, which would in general be expected to produce a bias in estimates of the age-atprimaparity (they would be over-estimated). However, we suggest that observation and marking techniques (and tag loss rates) were sufficiently consistent over time that general changes in the age at which animals were first re-sighted can be interpreted as indicating changes in the age at which animals were recruiting. The effect at the IoM appears clear, even though later cohorts are expected to be biased towards younger animals (and re-sighting effort has not decreased over the period of the study). Several observations support a delayed age of recruitment on the IoM: (1) in cohorts for which we have the most data, only a small proportion are seen breeding by age 5. This is not an artifact of marking or re-sightability as the double marked cohorts bear out, and (2) the almost complete absence of the later-marked cohorts, both at NR (1994-2005) and IoM (1997-2005), suggest that recruitment for these cohorts at IoM has barely begun. It is possible that increased population density at the IoM in recent years is linked to the decline in recruitment and the increased age of recruiting females. It is interesting to note that an increase in the age of recruiting seals also appears have taken place at the Sable Island seal colony in Atlantic Canada, as the population there has increased (Bowen et al., MS 2007, 2007).

On IoM, the numbers of females seen for the early cohorts approaches the expected number alive using the best estimates of survival and tag loss rates. This suggests that fidelity to the natal breeding site is high, at least for these cohorts. However recruitment at IoM appears to be in decline in later years, and there are occasional reports of IoM-tagged animals from other east coast sites such as Donna Nook, suggesting that one possible explanation for the decline is the emigration of seals from their natal colony to new breeding sites. In particular, a new colony has established at Fast Castle, close to the IoM (Fig. 5). The growth of Fast Castle has occurred at the same time that the IoM colony has begun to stabilise. Marked individuals, possibly from the IoM, have been seen at Fast Castle in recent years during late season ground counts (Duck and MacKey, MS 2008). Comprehensive re-sighting surveys of the Fast Castle and Donna Nook breeding colonies are needed to determine whether animals marked at IoM are responsible for this new expansion. By taking migration into account, it should be possible to estimate the 'true' number of seals recruiting to the North Sea breeding population, thus providing better parameter estimates for large-scale population models.

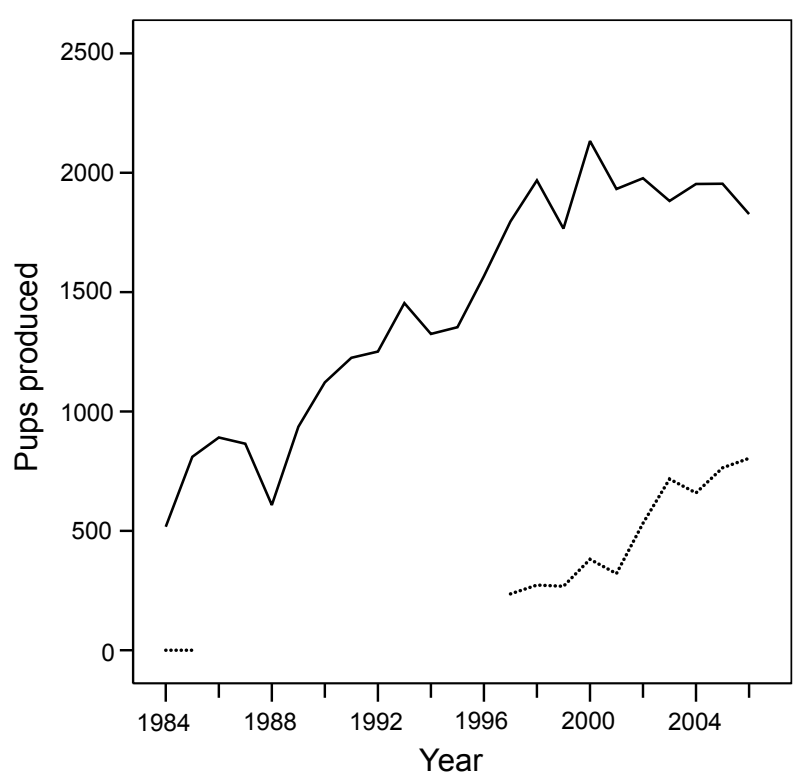

Fig. 5. Pup production are the Isle of May (solid line), compared with pup production at the nearby colony at Fast Castle (dashed line; Duck and MacKey, MS 2008).

\section{Acknowledgements}

The authors thank all those who helped with collecting these data in the field. The work received financial support from many sources, principally UK's NERC and EPSRC. Scottish Natural Heritage and Coastguard Agency provided assistance for fieldwork. All work was carried out under UK Home Office licence.

\section{References}

ALLEN, P.J., W. AMOS, P. P. POMEROY, and S. TWISS. 1995. Microsatellite variation in grey seals (Halichoerus grypus) shows evidence of genetic differentiation between two British breeding colonies. Mol. Ecol., 4: 653-662. doi:10.1111/j.1365-294X.1995.tb00266.x

BOWEN, W. D., J. I. MCMILLAN, D. LIDGARD, and W. BLANCHARD. MS 2007. Continued reduction in population growth rate of grey seals at Sable Island. CSAS Research Document, 2007/087, 20 p. http://www.dfo-mpo. gc.ca/csas/Csas/DocREC/2007/RES2007_087_e.pdf

BOWEN, W. D., J. I. MCMILLAN, and W. BLANCHARD. 2007. Reduced population growth of gray seals at Sable Island: evidence from pup production and age of primiparity. Mar. Mammal. Sci., 23: 48-64. doi:10.1111/ j.1748-7692.2006.00085.x

BOYD, I. L. 1985. Pregnancy and ovulation rates in grey seals (Halichoerus grypus) on the British coast. J. Zool., 205: 265-272.

BOYD, J. M., and R. N. CAMPBELL. 1971. The grey seal (Halichoerus grypus) at North Rona, 1959-1968. J. Zool., 164: 469-512. doi:10.1111/j.1469-7998.1971.tb01331.x

BUCKLAND, S. T., K. B. NEWMAN, C. FERNANDEZ, 
L. THOMAS, and J. HARWOOD. 2007. Embedding population dynamics models in inference. Stat. Sci., 22: 44-58. doi:10.1214/088342306000000673

DUCK, C. D., and B. L. MACKEY. MS 2005. Grey seal pup production in Britain in 2004. Annex II, No. 05/01. In: Scientific Advice on Matters Related to the Management of Seal Populations: 2005. SCOS Main Advice, p. 22-33. http://www.smru.st-andrews.ac.uk/documents/ SCOS_05_v2f.pdf

MS 2008. Grey seal pup production in Britain in 2007. SCOS Briefing Paper 08/1. In: Scientific advice on matters relating to the management of seal populations: 2008 . SCOS Main Advice 2008, SMRU, p. 31-42. http://www. smru.st-andrews.ac.uk/documents/SCOS_2008_v1.pdf

HALL, A. J., B. J. MCCONNELL, and R. J. BARKER. 2001. Factors affecting first-year survival in grey seals and their implications for life history strategy. J. Anim. Ecol., 70: 138-149. doi:10.1046/j.1365-2656.2001.00468.x

HARRISON, P., S. BUCKLAND, L. THOMAS, R. HARRIS, P. POMEROY, and J. HARWOOD. 2006. Incorporating movement into models of grey seal population dynamics. J. Anim. Ecol., 75: 634-645. doi: 10.1111/j.13652656.2006.01084.x

HARWOOD, J., S. S. ANDERSON, and M. G. CURRY. 1975. Branded grey seals at the Monach Isles, Outer Hebrides. J. Zool., 180: 506-508. doi:10.1111/j.1469-7998.1976. tb04698.x
HEWER, H. R. 1964. The determination of age, sexual maturity, longevity and the life table of the grey seal. Proc. Zool. Soc. Lond. A-GE, 142: 593-624.

KING, R., B. J. T. MORGAN, O. GIMENEZ, and S. P. BROOKS. 2009. Bayesian Analysis for Population Ecology. CRC Press, 456 p.

POMEROY, P., S. ANDERSON, S. TWISS, and B. MCCONNELL. 1994. Dispersion and site fidelity of breeding female grey seals on North Rona, Scotland. J. Zool., 233: 429-447. doi:10.1111/j.1469-7998.1994. tb05275.x

POMEROY, P., M. FEDAK, P. ROTHERY, and S. ANDERSON. 1999. Consequences of maternal size for reproductive expenditure and pupping success of grey seals at North Rona, Scotland. J. Anim. Ecol., 68: 235:253. doi:10.1046/ j.1365-2656.1999.00281.x

POMEROY, P., S. TWISS, and P. REDMAN. 2000. Philopatry, site fidelity and local kin associations within grey seal breeding colonies. Ethology, 106: 899-919. doi:10.1046/ j.1439-0310.2000.00610.x

SMOUT, S., P. POMEROY, and R. KING. 2010. Estimating demographic parameters for capture-recapture data in the presence of multiple mark types. Environ. Ecol. Stat., in press. 